\title{
CFD modelling of radioactive pollutants in a radiological laboratory
}

\author{
G. de With \\ Department of Radiation and Environment, \\ Nuclear Research \& Consultancy Group, Arnhem, The Netherlands
}

\begin{abstract}
An important aspect of indoor air quality is the presence of radioactive pollutants. These pollutants can be present in the form of gas or particles, and are typically found in nuclear installations and radiological laboratories. In this work the dispersion of radioactive pollutants in an indoor environment is studied using Computational Fluid Dynamics (CFD). The aim of this work is to evaluate the exposure to radioactive particles during an accidental release, and to evaluate suitable ventilation design to minimise exposure. These CFD findings are used towards improvement of the Dutch assessment procedures for evaluating the risk of radioactive exposure to radiological workers. For the purpose of this work a CFD model is developed to simulate the dispersion and nuclear decay of gas and aerosols, and the attachment and deposition of radioactive aerosols.
\end{abstract}

Keywords: CFD, radioactive pollution, particle modelling.

\section{Introduction}

Dispersion of radioactive pollutants in nuclear installations and radiological laboratories can form a potential health hazard to those people working nearby. The dispersion can come from an accidental release of radioactive gasses, or alternatively from a burst of radioactive dust particles. After release these pollutants are dispersed in the enclosed environment through the existing air recirculation. Consequently, this leads to an increase in radioactive concentrations in the humans' inhalation region, resulting in increased health risks.

The primary role of the laboratory ventilation system is to mitigate those health risks and minimise the exposure. Nevertheless, achieving an efficient 
ventilation system is a challenging task. At present most assessment procedures for air quality in nuclear laboratories are primarily based around some design values for the air exchange rate (AER). Nevertheless, varies studies have demonstrated that increasing the AER only has little effect on environmental conditions and exposure to pollutants [1]. More important is the air diffusion and air movement, which have considerably more impact on the ventilation performance. Consequently, the role of ventilation and its 3 dimensional flow characteristics must be an essential aspect in the air quality assessment. However, these 3 dimensional features are controlled through good ventilation design. To develop and optimise a good ventilation design faces design engineers with considerable challenges.

The purpose of this study is to investigate the dispersion of radioactive pollution using (Computational Fluid Dynamics) CFD computation. The computations take account of the dispersion and nuclear decay of gas and particles. In addition attachment of nuclear particles with aerosols and deposition of particles and aerosols are included. The algorithms for dispersion and nuclear decay of particles and gas are based on the work by Zhuo et al. [2]. The attachment of particles with aerosols and its deposition on the walls is based on the work by Porstendörfer [3] and Lai and Nazaroff [4].

This paper is organized in the following manner. In Section 2 a description of the CFD model is provided, followed by a validation (Section 3). Section 4 provides an overview of the CFD results, and the paper is finished with a summary of the conclusions (Section 5).

\section{Mathematical model equations}

As part of this investigation the CFD software FLUENT $^{\odot}$ is used. This section will provide a brief overview of the models that are used. Some of those models are already available in the CFD software and are used in the CFD computation where possible. However, algorithms to predict the deposition and dispersion of aerosols as well as the attachment of radio nuclides with the surrounding aerosols are developed in the framework of this study.

\subsection{Airflow modelling}

The basis for the CFD calculation is a set of two conservation equations. The first conservation equation refers to the conservation of mass and is defined in the following manner:

$$
\rho\left(\nabla \cdot u_{k}\right)=0
$$

Here $u$ is the velocity and $k$ is the index for the 3 velocity components. The second conservation equation is generally known as the Navier-Stokes equation. This equation describes the momentum conservation and is an implementation of Newton's second law applied to gas and liquid.

$$
\rho\left(\frac{\partial\left(u_{k}\right)}{\partial t}+\nabla \cdot\left(u_{k} u_{l}\right)\right)=-\nabla P+\nabla \cdot\left(\mu_{e f f} \nabla u_{k}\right)+S_{u, k}
$$


In this equation $u$ represents the velocity vector $(\mathrm{m} / \mathrm{s}), P$ is pressure, $\mu_{e f f}$ is the effective viscosity $\left(\mathrm{Ns} / \mathrm{m}^{2}\right), S_{u}$ is the source term and $\rho$ is the air density. The indices $k$ and $l$ are used to indicate the three velocity components. It is important to stress that most turbulent flow movements are not computed explicitly. Instead those flow movements are incorporated through an additional viscosity $\mu_{t}$. This concept is developed by Prantl around 1940 and is still adopted in most CFD studies. Therefore, the effective viscosity $\mu_{e f f}$ is the sum of both dynamic viscosity $\mu_{l}$ and turbulent viscosity $\mu_{t}$.

$$
\mu_{\text {eff }}=\mu_{l}+\mu_{t}
$$

For calculation of the turbulent viscosity the well-known $k-\varepsilon$ turbulence model is used.

\subsection{Modelling of gas dispersion}

For the dispersion of hazardous gas in the laboratory environment an additional conservation equation is applied. This conservation equation is shown below:

$$
\frac{\partial C_{m}}{\partial t}+\nabla \cdot u C_{m}=\nabla \cdot\left(\Gamma_{m} \nabla C_{m}\right)+S_{C, m}
$$

In this equation $C$ is the concentration of activity expressed in $\mathrm{Bq} / \mathrm{m}^{3}$ and $\Gamma$ is the diffusion coefficient $\left(\mathrm{m}^{2} / \mathrm{s}\right)$ of the hazardous gas. The two terms on the left hand side represent the convective transport of activity. On the right hand side the dispersion from diffusion is shown followed by the source term $S_{C}$. Where necessary, radioactive decay of the hazardous gas is incorporated in the source term $S_{C}$. Radioactive decay represents a sink to the activity of the gas and is therefore defined as $S_{C}=-\lambda C$. The diffusion coefficient $\Gamma$ in the dispersion equation is identical to the effective viscosity in the Navier-Stokes equation $\left(\mu_{e f f} / \rho\right)[5]$.

\subsection{Modelling of particle dispersion}

For the dispersion of particles a Eulerian based conservation equation is applied. The approach is based on the drift-flux method described by Lai and Nazaroff [4]. In literature the drift-flux method is described extensively and the method is also specifically developed for the dispersion of particles in an indoor environment [4-9].

In the drift-flux method the dispersion of particles is described by means of a continuity equation. This equation is comparable with the continuity equation for hazardous gas.

$$
\frac{\partial C_{m}}{\partial t}+\nabla \cdot\left[\left(u+v_{s, m}\right) C_{i}\right]=\nabla \cdot\left[\left(\Gamma_{m}+D_{m}\right) \nabla C_{m}\right]+S_{C, m} .
$$

In this equation $v_{s}$ is de terminal velocity $(\mathrm{m} / \mathrm{s})$ and $D$ is the Brownian diffusion coefficient of particles. The equation is comparable with eqn (4); 
however, there are some subtle differences. The convective transport is based on a modified flow field. In this flow field the terminal velocity of the particles is incorporated. In addition the Brownian diffusion coefficient is added to the effective diffusion. In this approach it is assumed that the particles do not influence the flow field significantly. For particles that normally do not exceed $10 \mu \mathrm{m}$ this is a very acceptable assumption.

\subsection{Deposition of particles}

The deposition of particles $J_{d}\left(\mathrm{~Bq} / \mathrm{m}^{2} / \mathrm{s}\right)$ on the surface is the product of particle concentration near the surface and the speed with which the particles are deposited. In mathematical terms this can be described as follows:

$$
J_{d}=v_{d} \cdot C_{b} .
$$

Here $v_{d}$ is the deposition velocity $(\mathrm{m} / \mathrm{s})$ of the particles and $C_{b}$ is the particle concentration in the vicinity of the surface $\left(\mathrm{Bq} / \mathrm{m}^{3}\right)$. Calculation of the deposition velocity is a difficult task. The deposition depends on a large number of factors including, gravitation, turbulence, thermal forces. In addition particles can be reentrained as a result of resuspention and rebound. It is therefore important to choose a deposition model that is suitable for indoor flow conditions. For this reason the deposition model of Lai and Nazaroff [4] is chosen. Their model is based on the experiments from Zhang et al. [10] and is developed specifically for deposition in the indoor environment.

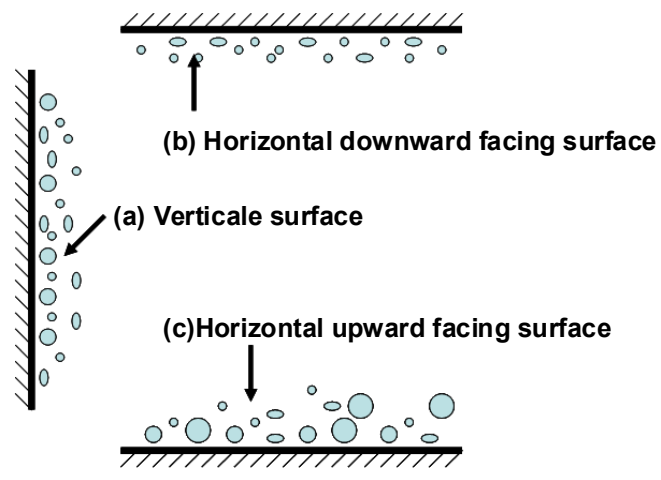

Figure 1: $\quad$ Orientation of the deposition surfaces.

The model equations from Lai and Nazaroff [4] describe the following three scenarios as shown in Figure 1:

- Deposition on vertical surfaces; these include the side walls.

- Deposition on horizontal surfaces facing downwards; these include the ceiling.

- Deposition on horizontal surfaces facing upwards; these include the floor.

Subject to orientation of the surface the smaller particles will deposit on the horizontal surfaces facing downwards and the vertical surfaces. The larger 
particles will deposit primarily on the horizontal surfaces facing upwards (Figure 1).

A summary of the model equations is presented in Table 1. In the equations the integral parameters $I, a$ and $b$ are used. Those parameters are based on experimental data and algebraic relations for idealized turbulent flows in the vicinity of the wall.

Table 1: $\quad$ Summary of the drift-flux deposition model.

\begin{tabular}{ll}
\hline \multicolumn{1}{c}{ Deposition speed } & Correlations \\
\hline \hline -Vertical surface & $v_{d v}=\frac{u^{*}}{I}$ \\
- Horizontal upward facing surface & $v_{d d}=\frac{v_{s}}{1-\exp \left(-\frac{v_{s} I}{u^{*}}\right)}$ \\
- Horizontal downward facing surface & $v_{d d}=\frac{v_{s}}{\exp \left(\frac{v_{s} I}{u^{*}}\right)-1}$ \\
\hline \hline
\end{tabular}

$$
\begin{aligned}
& I=\left[3,64 S c^{2 / 3}(a-b)+39\right] \\
& a=\frac{1}{2} \ln \left[\frac{\left(10,92 \cdot S c^{-1 / 3}+4,3\right)^{3}}{S c^{-1}+0,0609}\right]+\sqrt{3} \cdot \tan ^{-1}\left[\frac{8.6-10,92 \cdot S c^{-1 / 3}}{\sqrt{3} \cdot 10,92 \cdot S c^{-1 / 3}}\right] \\
& b=\frac{1}{2} \ln \left[\frac{\left(10,92 \cdot S c^{-1 / 3}+r^{+}\right)^{3}}{S c^{-1}+7,669 \cdot 10^{-4}\left(r^{+}\right)^{3}}\right]+\sqrt{3} \cdot \tan ^{-1}\left[\frac{2 r^{+}-10,92 \cdot S c^{-1 / 3}}{\sqrt{3} \cdot 10,92 \cdot S c^{-1 / 3}}\right]
\end{aligned}
$$

* Nomenclature: $S c=v_{l} D^{-1}, S c$ is the Schmidt number, $v_{l}$ de kinematic viscosity of air and $D$ is the diffusion coefficient of the particle; $r^{+}=d_{p} u^{*}\left(2 v_{l}\right)^{-1}, d_{p}$ is the diameter; $u^{*}$ is the friction velocity; $v_{s}$ is de terminal velocity of the particle.

The deposition velocity is calculated on the basis of the equations described in Table 1. The results are shown in Figure 2. The deposition velocity is presented for three different friction velocities $u^{*}$. A higher friction velocity is associated with higher wind speed and turbulent intensity in the near wall region.

The model assumes that the air velocities are sufficiently small that no resuspention or rebound of particles occurs [4]. Lai and Nazaroff [4] have indicated that modelling of resuspention and rebound is not essential due to low air velocities and turbulent intensities in the indoor environment. It is however important to note that implementation of a resuspention model in de drift-flux model is feasible [6].

\section{Model verification}

To validate the numerical model for simulating the indoor particle distribution, the measured data by Chen et al. [7] is adopted. Chen et al. [7] performed laboratory experiments for a model room with a geometry of $\mathrm{L}_{\text {ength }} \times \mathrm{W}_{\text {idth }} \times \mathrm{H}_{\text {eight }}=$ $0.8 \mathrm{~m} \times 0.4 \mathrm{~m} \times 0.4 \mathrm{~m}$. The inlet and outlet are of the same size $(0.04 \mathrm{~m} \times 0.04 \mathrm{~m})$ and 


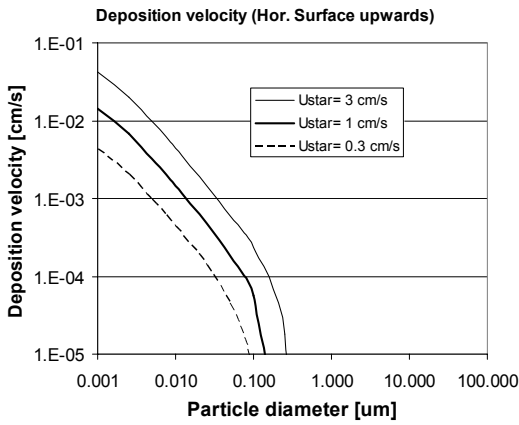

(a) Hor. downward facing surface

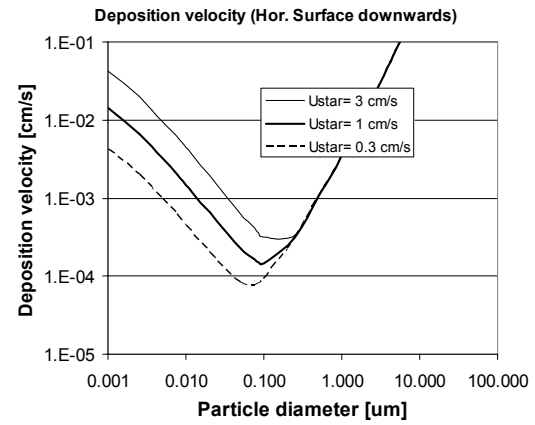

(b) Hor. upward facing surface

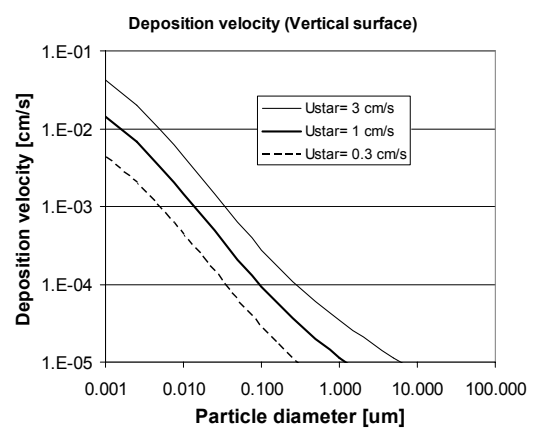

(c) Vertical surface

Figure 2: The deposition velocity as function of the diameter and the friction velocity. The calculations are based on an atmospheric pressure of 1 bar, temperature is $293 \mathrm{~K}$ and the density is $1000 \mathrm{~kg} / \mathrm{m}^{3}$.

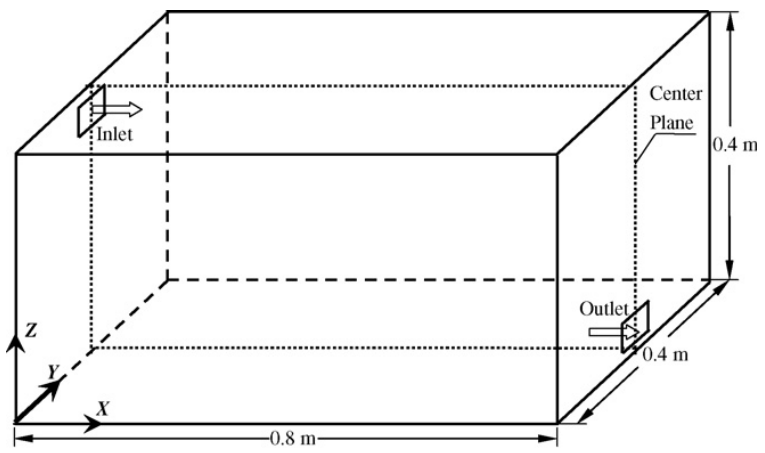

Figure 3: Schematic overview of the ventilation chamber for validation of the numerical model.

both are symmetrical with the center plane at $y=0.2 \mathrm{~m}$. A schematic overview of the model is shown in Figure 3. 
The imposed velocity at the inlet boundary is $0.225 \mathrm{~m} / \mathrm{s}$. At the same inlet boundary particles are injected and the particle concentration in the room is normalized with the concentration at the inlet. The particle density is $1400 \mathrm{~kg} / \mathrm{m}^{3}$ and the size of the particles is $10 \mu \mathrm{m}$. This particle size is suitable to validate the drift flux model as the drift flux is dominant for particles of this size. Chen et al. measured the airflow velocity and particle concentration with a Phase Doppler Anemometry (PDA) system. Picture (a) in Figure 4 shows a comparison of the simulated $\mathrm{x}$-velocity component with measured data. Comparison of the particle concentration is shown in picture (b) in Figure 4. The comparison is shown along the vertical z-axis at $x=0.4 \mathrm{~m}$ and $y=0.2 \mathrm{~m}$. The results show that both the airflow and particle concentration distribution is simulated accurately by the drift flux model.

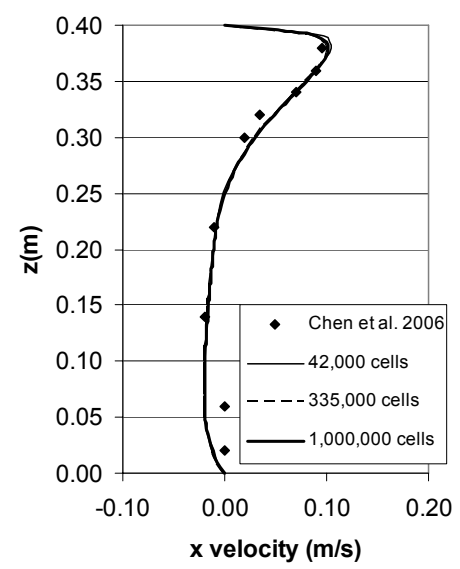

(a)

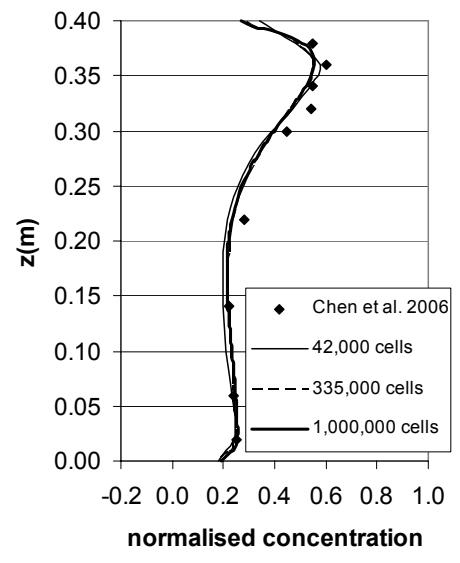

(b)

Figure 4: Comparison of the simulated airflow velocity and particle concentration distribution with the measured data of Chen et al. [7].

\section{Simulation results}

Simulations are performed for a medium size laboratory room of $\mathrm{L}_{\text {ength }} \times \mathrm{W}_{\text {idth }} \times \mathrm{H}_{\text {eight }}=6 \mathrm{~m} \times 6 \mathrm{~m} \times 3 \mathrm{~m}$. A workbench is located in the center of the laboratory and the pollution source is located directly above the workbench. The workbench is located in the center of the laboratory and is $\mathrm{L}_{\text {ength }} \times \mathrm{W}_{\text {idth }} \times \mathrm{H}_{\text {eight }}=$ $1 \mathrm{~m} \times 1 \mathrm{~m} \times 1 \mathrm{~m}$. In this work a release of radioactive particles is studied. The particles' radioactivity is long-lived providing a direct relation between the concentration of particles and the exposure to radioactivity. Varies particle diameters are evaluated in combination with different ventilation scenarios. Two flow features are assessed in each simulation. They include the mean concentration of particles at $1.5 \mathrm{~m}$ above ground and the particle concentration 
directly above the release at $1.5 \mathrm{~m}$ above ground. Both features highlight the exposure to humans and the ability of the ventilation system to minimize particle concentration in the area of interest. The height above ground of $1.5 \mathrm{~m}$ is based on the average height of human's inhalation, and provides a best indicator for the human's internal exposure to aerosols.

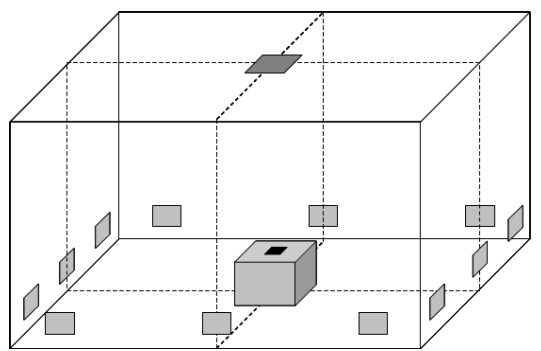

(a)

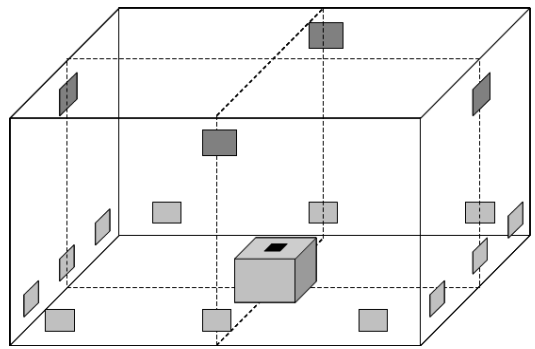

(c)

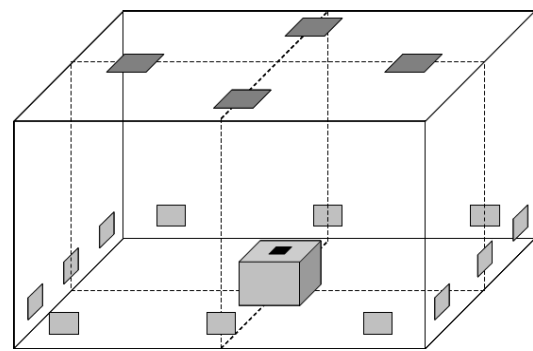

(b)

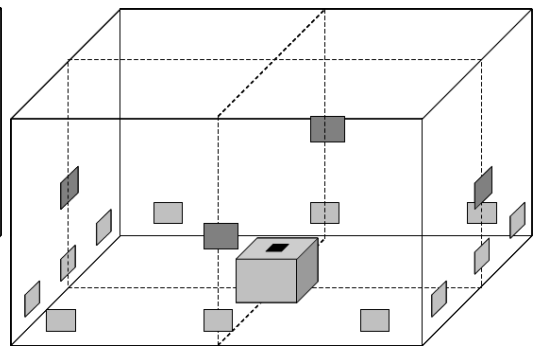

(d)

Figure 5: Laboratory ventilation scenarios selected for simulation. The 12 inlet ventilation slots, highlighted in light grey, are located at the bottom of the side walls. The pollution source is above the table and indicated by a small black surface. The ventilation extractors are highlighted in dark grey and vary for each scenario.

The concentration levels are expressed relative to the concentration at the release. In addition it is important to note that coagulation of the radioactive particles with the surrounding aerosols is not selected. The four ventilation scenarios that are selected are shown in Figure 5. All ventilation scenarios are equipped with 12 ventilation slots $(0.1 \mathrm{~m} \times 0.1 \mathrm{~m})$ at the bottom of the four side walls. Location of the ventilation extractors varies for each ventilation scenario. In addition the effect from air exchange is studied and the air exchange varies from $2 \mathrm{hr}^{-1}$ to $10 \mathrm{hr}^{-1}$.

The simulation results are presented in Figure 6 and Figure 7. The first figure contains two pictures and shows the particle concentration directly above the release. The second figure contains two pictures as well and shows the mean particle concentration in the laboratory at $1.5 \mathrm{~m}$ above ground.

Picture (a) of Figure 6 shows a number of interesting phenomena. For all three ventilation cases, the particle concentration reduces as the particle diameter 
increases. However, for smaller particles up to $5 \mu \mathrm{m}$ an increase in the ventilation's air exchange rate reduces the particle concentration, while for particles larger then $5 \mu \mathrm{m}$ there is an opposite effect. For larger particles the concentration above the release reduces rapidly. This is partly due to the large gravitational forces that result in considerable deposition. However, when ventilation is increased the larger particles are less prone to deposition and are instead entrained into the main flow stream. As a result there is an increase in particle concentration. A further phenomenon is the diminishing effect from additional ventilation above 5 AER for particles up to $5 \mu \mathrm{m}$. This phenomenon has been reported in varies studies [1] and is also confirmed in this work.

The simulation results from scenario $B, C$ and $D$ are shown in picture $(b)$ of Figure 6. The three ventilation cases suggest that the location of the ventilation extractors have limited effect on the particle concentration near the release. In contrast with the findings from scenario A, scenario B to D have no negative adverse effects when the AER is increased.

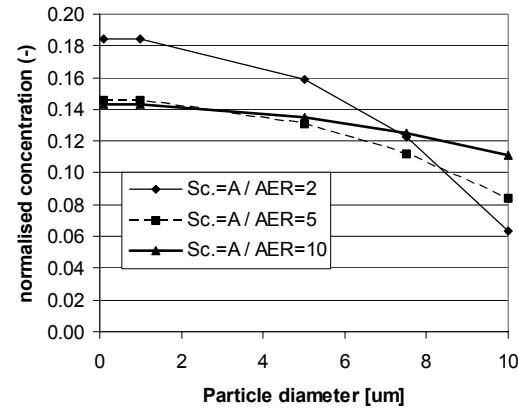

(a)

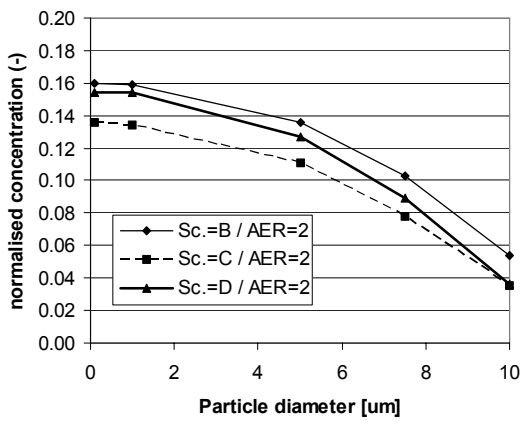

(b)

Figure 6: Particle concentration above the release at $1.5 \mathrm{~m}$ above ground level.

Figure 7 shows the mean particle concentration in the laboratory at $1.5 \mathrm{~m}$ above ground. In this figure a distinct difference between scenario A (picture a) and the other scenarios B to D (picture b) is shown. Where ventilation is applied directly above the release (scenario A) concentration levels are more than ten times smaller when compared against the other three ventilation scenarios. Similar to Figure 6 the results from scenario A also show some adverse effects from increased ventilation for particles of around $10 \mu \mathrm{m}$. The results clearly suggest that the scenarios $\mathrm{B}$ to $\mathrm{D}$ have comparable ventilation features. In contrast scenario A shows features that are typically found in a fume hood.

For particles smaller than $1 \mu \mathrm{m}$ concentration levels in the laboratory are unaffected by the particle diameter. For those types of particles the terminal velocity becomes insignificant in a well ventilated room with more then 2 air exchanges per hour. 
At this stage more simulations are required for different ventilation arrangements and laboratory setup before general conclusions can be drawn. However, the above findings provide some first data to review existing assessment procedures for air quality in radiological laboratories.

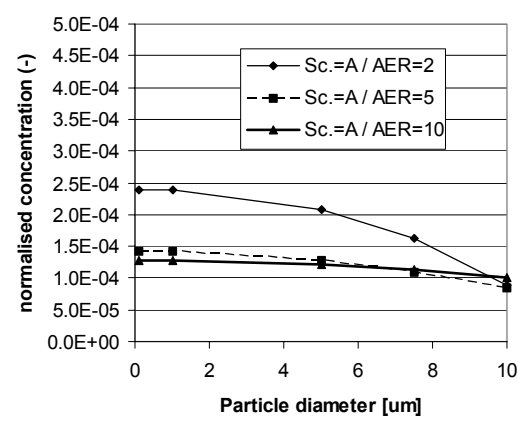

(a)

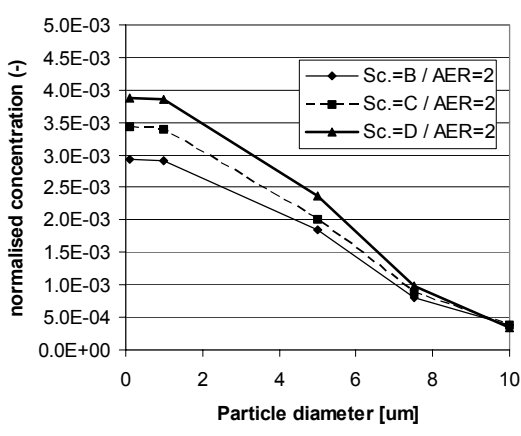

(b)

Figure 7: Mean particle concentration in the room at $1.5 \mathrm{~m}$ above ground level.

\section{Conclusions}

The conclusions of this work are as follows:

- A CFD model is developed to predict the dispersion and decay of radioactive gasses and particles. Particle deposition on the surfaces and effects from gravitational settling are accounted for.

- The prediction of particle dispersion is validated against experimental data of Chen et al. [7].

- A total of four ventilation scenarios are investigated and in addition the effects from increased air exchange are studied. The simulation results suggest the following:

o In scenario A an increase in ventilation has a negative adverse effect on the particle concentration for particles large then $10 \mu \mathrm{m}$.

o The dispersion of particles in scenario $\mathrm{B}$ to $\mathrm{D}$ is comparable. In contrast the particle dispersion in scenario A is more similar with the dispersion found in fume hoods.

o The results suggest concentration levels for particles smaller then $1 \mu \mathrm{m}$ are unaffected by the particle diameter.

- Suggestions for further work are to take account of induced turbulence from laboratory workers and thermal driven flows. Both flow features enhance mixing and may affect concentration levels in the laboratory. 


\section{References}

[1] Crane J., Ellis I., Siebers R., Grimmet D., Lewis S., Fitzharris P., A pilot study of the effect of mechanical ventilation and heat exchange on housedust mites and Der p 1 in New Zealand homes. Allergy, Vol. 53, No 8, 755$762,1994$.

[2] Zhuo W., Iida T., Moriizumi J., Aoyagi T., Takahashi I., Simulation of the concentrations and distributions of indoor radon and thoron. Rad. Prot. Dosim., Vol. 93 No 4, pp 357-368, 2001.

[3] Porstendörfer J., Properties and behaviour of radon and thoron and their decay products in the air. Proc. Fifth Int. Symp. on the Natural Radiation Environment. EU commission, Luxembourg, 2003.

[4] Lai A.C.K., Nazaroff W.W., Modelling indoor particle deposition from turbulent flow onto smooth surfaces. J. Aerosol Sci., Vol. 31, No 4, pp. 463-476, 2000.

[5] Hinze J.O., Turbulence. $2^{\text {nd }}$ Edition, McGraw-Hill, New York, 1975.

[6] Schneider T., Kildesø J., Breum N.O., A two compartment model for determining the contribution of sources, surface deposition and resuspention to air and surface dust concentration levels in occupied rooms. Build. Environ., Vol. 34, pp. 583-595, 1999.

[7] Chen F., Yu S.C.M., Lai A.C.K., Modelling particle distribution and deposition in indoor environments with a new drift-flux model. Atmospheric Environ., Vol. 40, pp. 357-367, 2006.

[8] Gao N.P., Niu J.L., Modelling particle dispersion and deposition in indoor environments. Atmospheric Environ., Vol.41, pp. 3862-3876, 2007.

[9] Zhao B., Wu J., Particle deposition in indoor environments: Analysis of influencing factors. J. Hazard. Mat., Vol. 147, pp. 439-448, 2007.

[10] Zhang J.S., Shaw C.Y., Nguyen-Thi L.C., MacDonald R.A., Kerr G., Field measurements of boundary layer flows in ventilated rooms. ASHRAE Trans., Vol. 101, Part2, pp. 116-124, 1995. 